\title{
Alteraciones polisomnográficas en lactantes con reflujo gastroesofágico
}

\author{
Paul Harris D, Pablo Brockmann Va, Carla Muñoz $0^{a}$, \\ Sebastián Mobarec Kă, Tomás Mesa L, Ignacio Sánchez D. \\ Polysomnographic abnormalities in \\ infants with gastroesophageal reflux \\ disease
}

Background: Infant apnea is a common problem that conveys significant burden to families and physicians. Its temporal relationship with gastroesophageal reflux (GER) is controversial. Aim: To establish whether infants with GER of different magnitude have a higher incidence of respiratory events than children without GER. Patients and Methods: 146 consecutive patients were evaluated with polisomnography (PSG) and with an esophageal $\mathrm{pH}$ probe. Those infants without GER episodes or with an esophageal $\mathrm{pH}$ below 4.0 in less than $5 \%$ of total sleep time were considered as physiologic GER (Group I), between 5-10\% as mild GER (Group II), and over 10\% as severe GER (Group III). These groups were evaluated for demographic and polysomnographic characteristics. Results: There were no differences in the demographic and global PSG characteristics neither in oxymetry, heart rate or electroencephalographic abnormalities. Group III infants had a higher percentage of active sleep compared to infants of Group I ( $\mathrm{p}<0.05$ ) and higher incidence of central pauses and apneas compared to infants of Group I ( $p<0.05$ ). Conclusions: The presence of severe GER may modify the sleep pattern in infants, increasing the percentage of active sleep. The presence of central pauses and apneas in infants with severe GER suggest an association between the inmaturity of the respiratory centers and the adaptation of the anti-reflux mechanisms (Rev Méd Chile 2003; 131: 1143-50).

(Key Words: Gastroesophageal reflux, Polysomnography; Sleep disorders)

Recibido el 29 de noviembre, 2002. Aceptado en versión corregida el 5 de agosto, 2003. Secciones de Gastroenterología, Neurología y Respiratorio, Departamento de Pediatría, Escuela de Medicina, Pontificia Universidad Católica de Chile, Santiago, Chile.

anternos de Medicina, Pontificia Universidad Católica de Chile.

$\mathrm{L}^{2}$ a relación entre reflujo gastroesofágico (RGE) $y$ apnea en los lactantes ha sido controversial ${ }^{1-6}$. Aunque se ha sugerido una relación entre ambos, tanto el RGE como la apnea disminuyen con la

Correspondencia a: Paul Harris D. Lira 85, $5^{\text {to }}$ piso, Santiago, Chile. Fono: 354 6843; Fax: 638 4307. E mail pharris@med.puc.cl edad, dificultando un análisis causa efecto ${ }^{4}$. Mientras la apnea del lactante puede ser un evento que pone en riesgo vital al niño, el RGE es un evento común y fisiológico en la mayoría de los lactantes. Si bien el RGE puede ser la causa primaria de apnea y otros desórdenes respiratorios, la evidencia publicada en la literatura revela que el RGE es más bien una causa inusual de apnea obstructiva ${ }^{3-6}$. Del 
mismo modo, los episodios de apnea (particularmente los de tipo obstructivo) pueden inducir el $\mathrm{RGE}^{7-10}$.

Con la introducción de la polisomnografía (PSG) nocturna extendida con medición simultánea del $\mathrm{pH}$ esofágico, para el estudio de los eventos respiratorios, la asociación entre RGE y apnea ha sido reevaluada. Numerosos estudios han intentado establecer una relación temporal causa-efecto, entre eventos de $\mathrm{pH}$ ácido y eventos respiratorios tipo apnea. Dichos estudios han sido controversiales y han fallado en demostrar una relación consistente causa-efecto. Reportes de nuestro propio grupo han mostrado que esta relación cualitativa es más bien esporádica ${ }^{11}$.

Los objetivos de este estudio fueron establecer si aquellos lactantes que presentan RGE en diferentes rangos, de acuerdo a los criterios pHmétricos habituales para pHmetrías extendidas de $24 \mathrm{~h}$, presentan una mayor frecuencia de eventos respiratorios que los niños sin RGE considerando dichos criterios. Nosotros planteamos que si bien la relación cualitativa entre evento de reflujo y evento respiratorio es difícil de demostrar por la naturaleza aislada del fenómeno, el rol genuino de la exposición ácida al esófago mantenido puede ser visualizado en la magnitud de los eventos respiratorios en dichos pacientes.

\section{PACIENTES Y MÉTODOS}

Pacientes. En un modelo prospectivo, se incluyeron 146 pacientes consecutivos desde mayo de 1997 hasta mayo de 2002 con consentimiento informado por sus padres o guardianes. Los pacientes fueron referidos al centro de estudios médicos del sueño de la Pontificia Universidad Católica de Chile para PSG con pHmetría simultánea. Los pacientes fueron enrolados con un patrón consecutivo, sin excluir ningún paciente excepto aquellos en los cuales el PSG fue requerido sin pHmetría por su médico tratante. El centro de estudios médicos del sueño realiza PSG con pHmetría simultánea sólo cuando ésta es requerida por el médico tratante.

PSG. Los lactantes se sometieron a un PSG con pHmetría con un polosomnógrafo ALCE 3.0 (versión $1.20 \mathrm{H}$, Healthdyn Respironics Marietta GA,
USA). Las siguientes variables fueron registradas en forma continua: electroencefalograma (8 canales), oculograma ( 2 canales) y electromiograma (1 canal en el mentón), flujo aéreo nasal por termistor (1 canal), movimientos torácicos y abdominales (2 canales), electrocardiograma (1 canal), saturación de oxígeno (1 canal), onda de pulso (1 canal), movimientos corporales por inductancia pisoeléctrica (1 canal), frecuencia cardíaca (1 canal), posición corporal (1 canal) y presencia de luz en la sala (1 canal). El tiempo de respuesta para todas las variables fue no mayor a 0,5 s para las señales respiratorias. Los registros se realizaron por períodos de 8 a $10 \mathrm{~h}$ en cada paciente. Cada lactante se estudió en una habitación separada con una temperatura ambiental estable. Uno de los padres acompañó al lactante durante el estudio. Los registros fueron hechos durante la noche en todo lactante mayor de 3 meses de edad y fue opcional realizar el estudio en forma diurna o nocturna en los lactantes menores de 3 meses. El horario y tipo de alimentación no se modificó durante este estudio. La eventual terapia anti-reflujo se discontinuó $72 \mathrm{~h}$ antes del estudio en aquellos lactantes con terapia médica. Un tecnólogo experimentado en PSG realizó y registró el estudio.

pHmetría. La sonda de $\mathrm{pH}$ constó de un electrodo que fue ubicado en el tercio distal del esófago como ha sido previamente descrito ${ }^{12}$ por un gastroenterólogo pediátrico. Se utilizaron sondas desechables de poliuretano con electrodos de antimonio (Sandhill Scientific, Inc., CO, USA) con tiempos de respuesta de $4 \mathrm{~s}$. Se realizó una calibración individual antes de cada estudio, usando soluciones de referencia con $\mathrm{pH} 1,0$ y 7,0. Cuando la posición de la sonda no fue satisfactoria con las técnicas rutinarias de posicionamiento, se obtuvo una radiografía de tórax para documentar y eventualmente corregir la posición de la sonda de $\mathrm{pH}^{13}$, lo cual ocurrió en un paciente.

Grupos en estudio. Para la constitución de los grupos se utilizaron las definiciones de reflujo previamente establecidas por las sociedades internacionales ${ }^{14}$. Un episodio de reflujo ácido se definió como la caída de $\mathrm{pH}$ menor a 4 por más de 6 s. Como definiciones operacionales, aquellos lactantes que no presentaron ningún episodio de RGE o que presentaron un porcentaje de tiempo total con pH menor a 5\% del tiempo total de sueño, 
fueron considerados como portadores de un RGE fisiológico (Grupo I). Aquellos lactantes con un porcentaje de tiempo total con $\mathrm{pH}$ menor a 4 , entre 5 y $10 \%$ del tiempo total de sueño fueron considerados portadores de un RGE moderado (Grupo II). Aquellos lactantes con un porcentaje de tiempo total con $\mathrm{pH}$ menor a 4 , mayor a $10 \%$ del tiempo total de sueño fueron considerados como portadores de un RGE intenso (Grupo III). La magnitud del descenso del pH no se consideró en estas definiciones operacionales ${ }^{14}$.

Puntaje y definiciones de PSG: Dos investigadores experimentados realizaron la puntuación de los estudios de PSG. Una vez que los registros fueron analizados, un tercer médico (gastroenterólogo), analizó los resultados de $\mathrm{pH}$ de acuerdo a las definiciones previamente descritas. Apnea central fue definida como el cese de la respiración que dura más de $20 \mathrm{~s}$, sin esfuerzo respiratorio, mientras que pausa central fue definida como el cese de la respiración por más de $6 \mathrm{~s}$ (en niños mayores de 1 año, pausa central fue definida como el cese de la respiración por más de 10 pero menos de $20 \mathrm{~s}$ ). Apnea obstructiva fue definida como el cese de la respiración por más de $10 \mathrm{~s}$ con esfuerzo respiratorio y pausa obstructiva cuando duraba más de 3 pero menos de $10 \mathrm{~s}$. Apnea mixta fue definida como el cese de la respiración que dura más de $10 \mathrm{~s}$, inicialmente sin esfuerzo respiratorio seguido por esfuerzo respiratorio. El índice de apnea se definió como el número de apneas por hora e índice de pausas se definió como el número de pausas por hora, de acuerdo a criterios publicados ${ }^{15-17}$. La respiración periódica fue considerada normal hasta el 5\% del tiempo total de sueño en recién nacidos y hasta el $1 \%$ del tiempo total de sueño en lactantes de 6 meses, como fue definido por Shannon y cols ${ }^{18}$.

Estadística. Las comparaciones entre grupos se relizaron con la prueba $t$ de Student y ANOVA para variables continuas. Significancia estadística se definió como un valor $\mathrm{p}$ menor a 0,05.

\section{RESULTADOS}

Conformación de los grupos. Se enrolaron 146 niños para este estudio: 85 niños presentaron un reflujo en rango fisiológico (Grupo I), 21 en rango moderado (Grupo II) y 40 lactantes en rango intenso (Grupo III). Debido a que el pH varió entre los diferentes estadios de sueño (vigilia, sueño activo, sueño quieto y sueño indeterminado), se consideraron los porcentajes de $\mathrm{pH}$ menor a 4 más elevados, independiente del estadio de sueño (Figura 1). En los 3 grupos el porcentaje

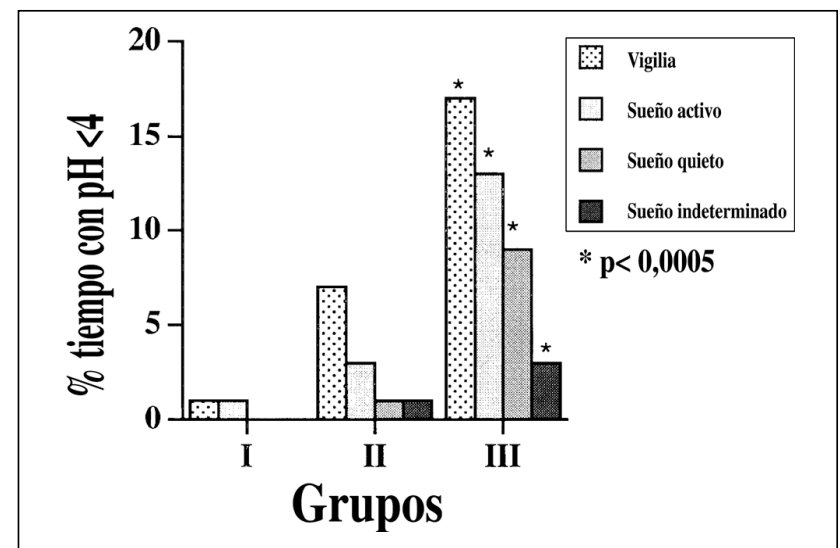

FiguRA 1. Porcentaje del estudio con pH menor a 4.0 en vigilia, sueño activo, sueño quieto y sueño indeterminado, en lactantes sometidos a PSG con pHmetría, de acuerdo a intensidad del RGE. Debido a que el $\mathrm{pH}$ varió entre los diferentes estadios de sueño, se consideraron los porcentajes de pH menor a 4,0 más elevados, independiente del estadio de sueño, para clasificar los pacientes en 3 grupos. En los 3 grupos el porcentaje máximo de $\mathrm{pH}<4,0$ dentro del rango de definición, se presentó en vigilia y en sueño activo, disminuyendo en fases de sueño quieto o indeterminado. Los lactantes del Grupo III presentaron un mayor índice de reflujo en todos los estadios de sueño $(p<0,0005)$. 
máximo de $\mathrm{pH}<4,0$ dentro del rango de definición, se presentó en vigilia y en sueño activo, disminuyendo en fases de sueño quieto o indeterminado. Como era esperado, los lactantes del Grupo III presentaron un mayor índice de reflujo en todos los estadios de sueño ( $p<0,0005)$.

Características demográficas. Las características demográficas de los tres grupos aparecen descritos en la Tabla 1. No hubo diferencia significativa en ninguna de las variables analizadas, lo cual anula el efecto prematurez y peso de los lactantes para las interpretaciones posteriores.
PSG. Los resultados generales del PSG, incluyendo los tiempos de registro y eficiencia de sueño, aparecen descritos en la Tabla 2. La eficiencia del sueño fue de $82,3 \%$ en promedio, y no hubo diferencia significativa entre los 3 grupos para las variables descritas. La arquitectura del sueño varió entre los grupos, encontrándose una mayor proporción de sueño activo y una menor proporción de sueño quieto en el Grupo III comparado con el Grupo I ( $\mathrm{p}<0,05)$ (Figura 2). La presencia de respiración periódica y las alteraciones de la oximetría aparecen descritas en la Tabla 3. La saturación arterial de oxígeno promedio fue $96,5 \%$. La satura-

Tabla 1. C aracterísticas demográficas por grupos

\begin{tabular}{|c|c|c|c|c|c|}
\hline & Grupo I & Grupo II & Grupo III & Total & Valor $\mathrm{p}$ \\
\hline № & 85 & 21 & 40 & 146 & ns \\
\hline Niños/niñas & $46 / 39$ & $8 / 13$ & $24 / 16$ & $78 / 78$ & ns \\
\hline RN pretérmino & 21 & 5 & 14 & 40 & ns \\
\hline Edad, meses (X $\pm \mathrm{DS})$ & $2,9 \pm 2,7$ & $2,8 \pm 2,7$ & $2,6 \pm 2,6$ & $2,8 \pm 2,6$ & ns \\
\hline 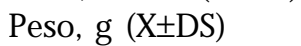 & $4964 \pm 2008$ & $4982 \pm 2087$ & $4682 \pm 3535$ & $4893 \pm 2057$ & ns \\
\hline 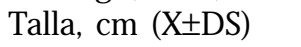 & $56 \pm 8$ & $54,6 \pm 6$ & $55,6 \pm 13$ & $55 \pm 8$ & ns \\
\hline
\end{tabular}

Tabla 2. C aracterísticas generales del PSG por grupos

\begin{tabular}{|c|c|c|c|c|c|}
\hline & $\begin{array}{c}\text { Grupo I } \\
\mathrm{n}=85\end{array}$ & $\begin{array}{c}\text { Grupo II } \\
\mathrm{n}=21\end{array}$ & $\begin{array}{c}\text { Grupo III } \\
n=40\end{array}$ & $\begin{array}{c}\text { Total } \\
\mathrm{n}=146\end{array}$ & $\begin{array}{c}\text { Valor } \\
\mathrm{p}\end{array}$ \\
\hline PSG nocturno (\%) & $49(58)$ & $13(62)$ & $20(50)$ & $82(56)$ & ns \\
\hline Tiempo registro, $\min (\mathrm{X} \pm \mathrm{DS})$ & $457 \pm 72$ & $478 \pm 53$ & $450 \pm 56$ & $458 \pm 66$ & ns \\
\hline Eficiencia del sueño, \% (X士DS) & $82,7 \pm 8$ & $81,4 \pm 10$ & $81,7 \pm 8$ & $82,3 \pm 8$ & ns \\
\hline
\end{tabular}

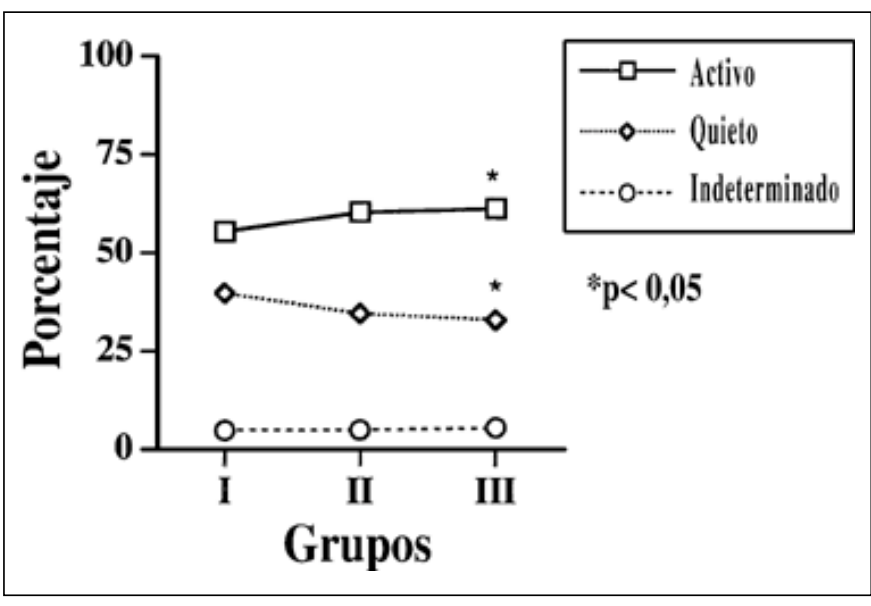

FIgURA 2. Arquitectura del sueño según Grupos de estudio. Los estadios de sueño se expresaron como porcentajes. La arquitectura varió entre los grupos, encontrándose una mayor proporción de sueño activo y una menor proporción de sueño quieto en el Grupo III comparado con el Grupo I ( $p$ $<0,05)$. 
ción de oxígeno en ausencia de eventos respiratorios varió entre 84,7 y $98,5 \%$ para todos los grupos. Sin embargo, la desaturación máxima relacionada a eventos respiratorios fue 86,2 y 78,9\%, en relación a pausas y apneas, respectivamente. La frecuencia cardíaca basal fue 131 latidos por minuto. No hubo diferencia significativa entre los 3 grupos en ninguna de las variables descritas.

Las características de las apneas y pausas aparecen descritas en la Tabla 4. El grupo con

Tabla 3. Eventos generales

\begin{tabular}{|lccccc|}
\hline Evento & $\begin{array}{c}\text { Grupo I } \\
\mathrm{n}=85\end{array}$ & $\begin{array}{c}\text { Grupo II } \\
\mathrm{n}=21\end{array}$ & $\begin{array}{c}\text { Grupo III } \\
\mathrm{n}=40\end{array}$ & $\begin{array}{c}\text { Total } \\
\mathrm{n}=146\end{array}$ & Valor $\mathrm{p}$ \\
\hline $\begin{array}{l}\text { Respiración periódica, \%TST* } \\
\text { Oximetría (X+DS): }\end{array}$ & $4,2 \pm 8$ & $3,9 \pm 6$ & $3,4 \pm 7$ & $3,9 \pm 7$ & $\mathrm{~ns}$ \\
$\quad$ - \% saturación O2 & $96,4 \pm 4$ & $97 \pm 2$ & $96,3 \pm 2$ & $96,5 \pm 3$ & $\mathrm{~ns}$ \\
$\quad$ - mínima saturación O2 & $84,6 \pm 9$ & $85,9 \pm 10$ & $84,1 \pm 10$ & $84,7 \pm 9$ & $\mathrm{~ns}$ \\
$\quad$ - máxima saturación O2 & $98,6 \pm 3$ & $99 \pm 1$ & $98,2 \pm 2$ & $98,5 \pm 2$ & $\mathrm{~ns}$ \\
$\quad$ - \% desaturación con pausas & $85,9 \pm 7$ & $88,1 \pm 6$ & $85,9 \pm 7$ & $86,2 \pm 7$ & $\mathrm{~ns}$ \\
$\quad$ Frecuencia cardíaca: & $79,8 \pm 14$ & $75,5 \pm 20$ & $79,6 \pm 17$ & $78,9 \pm 16$ & $\mathrm{~ns}$ \\
$\quad$ - promedio, lpm^ (X+DS) & $136 \pm 13$ & $129 \pm 25$ & $123 \pm 25$ & $131 \pm 19$ & $\mathrm{~ns}$ \\
$\quad$ Electroencefalograma: & $107-163$ & $117-150$ & $115-138$ & $111-155$ & $\mathrm{~ns}$ \\
$\quad$ - normal, No (\%) & 81 (95) & $20(95)$ & $35(87,5)$ & $136(93)$ & $\mathrm{ns}$ \\
\hline
\end{tabular}

*TST, tiempo total de sueño; ^lpm, latidos por minuto

Tabla 4. Eventos respiratorios

\begin{tabular}{|lcccc|}
\hline Eventos por hora & $\begin{array}{c}\text { Grupo I } \\
\mathrm{n}=85\end{array}$ & $\begin{array}{c}\text { Grupo II } \\
\mathrm{n}=21\end{array}$ & $\begin{array}{c}\text { Grupo III } \\
\mathrm{n}=40\end{array}$ & $\begin{array}{c}\text { Total } \\
\mathrm{n}=146\end{array}$ \\
\hline Indice de apnea: & & & & \\
$\quad$ - central & 0,0 & 0,0 & $0,1 *$ & 0,4 \\
- obstructiva & 1,0 & 0,7 & 0,3 & 1,0 \\
- mixta & 1,2 & 0,2 & 1,2 & 0,7 \\
Indice de pausas: & & & & \\
- central & 8,2 & 8,7 & $11,3 *$ & 9,2 \\
- obstructiva & 1,7 & 1,2 & 1,4 & 1,2 \\
- mixta & 0,6 & 0,5 & $2,7 *$ & 1,6 \\
Pausas por estadio de sueño: & & & & \\
- activo & 16,1 & 14,3 & 18,6 & 16,5 \\
- quieto & 6,6 & 6,7 & 10,1 & 7,6 \\
- indeterminado & 9,9 & 7,7 & 11,2 & 9,9 \\
Tipo de pausas: & & & & \\
- rango, seg & $3,2-8,9$ & $2,3-9,7$ & $3,1-8,9$ & $2,3-8,9$ \\
- centrales, \% & 88,8 & 22,8 & 88,5 & 89,0 \\
- obstructivas, \% & 5,7 & 1,9 & 6,8 & 5,8 \\
- mixtas, \% & 9,5 & 11,5 & 10,9 & 9,6 \\
\hline
\end{tabular}

$* p<0,05$ comparado con Grupo I. 
mayor RGE (Grupo III) presentó un mayor índice de apneas centrales ( $p<0,05)$, un mayor índice de pausas centrales $(p<0,05)$, y un mayor índice de pausas mixtas ( $p<0,05$ ), comparado con el grupo de RGE fisiológico (Grupo I). Los eventos respiratorios en los lactantes durante estos estudios se resolvieron con estimulación física. Maniobras cardiopulmonares no fueron necesarias.

\section{Discusión}

La relación entre RGE y apnea ha sido largamente debatida en la literatura. Si bien, el RGE fisiológico está presente en 25 a $50 \%$ de los lactantes normales ${ }^{19}$, es la presencia de complicaciones y no la magnitud del vómito o regurgitación, lo que define al RGE como patológico. Las presentaciones clínicas del RGE en lactantes incluyen la regurgitación, vómitos, irritabilidad, dolor abdominal y alteraciones del sueño. Las complicaciones establecidas del reflujo pueden ser agrupadas en 3 variedades: complicaciones gastrointestinales (esofagitis, estenosis, esófago de Barrett), mal incremento de peso y complicaciones pulmonares secundarias ya sea a una aspiración directa (neumonía aspirativa, apnea obstructiva) o a mecanismos reflejos (laringoespasmo, apnea central u obstructiva). La plausibilidad biológica en la relación temporal entre apnea y reflujo está fundamentada por diferentes mecanismos, directos e indirectos (reflejo de broncoconstricción, reflejo esofágico abductor, reflejo quemolaríngeo) ${ }^{20-22}$. Aunque hay evidencias que apoyan una asociación entre RGE y apnea, el estudio más apropiado para estudiar esta relación no ha sido establecido. La pHmetría de $24 \mathrm{~h}$ es un procedimiento bien estandarizado en términos de indicación, ejecución y análisis ${ }^{12,13}$, sin embargo, no es tan preciso en términos de estudiar la relación entre cambios de $\mathrm{pH}$ con otras variables biológicas. Aun así, desde un punto de vista teórico, la PSG con pHmetría simultánea debería ser el método de elección para estudiar lactantes con apnea del sueño. Un aspecto limitante en este análisis es la ausencia de parámetros normales para este último estudio. Por esta razón nosotros escogimos los parámetros normales o habituales de una pHmetría de $24 \mathrm{~h}$ para analizar los resultados en el presente trabajo ${ }^{14}$.
Aunque hay evidencia biológica que apoya una relación entre apnea y RGE, la evidencia clínica que apoya esta asociación es aún controversial $^{5,21-23}$. Es probable que un registro de tiempo breve, como es una noche, puede perder eventos clínicamente significativos que ocurran con una frecuencia menor. Aunque no hay solución para este problema, debido a que la PSG prolongada ambulatoria con pHmetría incluida no está disponible actualmente, tal limitación es común para todos los estudios que utilizan esta técnica.

En unos de estos estudios que evaluaron apneas y manifestaciones clínicas de RGE, el riesgo de apnea por regurgitación o vómito fue mayor en lactantes con RGE. Muy pocos eventos apneicos fueron secundarios a RGE ${ }^{24}$. En términos de apnea y reflujo oculto, ha habido un énfasis significativo en modelos animales que apoyan este tema ${ }^{25,26}$. Sin embargo, en la medida que la evidencia experimental de modelos animales ha sido trasladada a humanos, esta relación ha sido controversial ${ }^{27}$ y la asociación parece más bien ser coincidente en lactantes estudiados sin PSG ${ }^{21,28}$. Aquellos estudios que examinan la frecuencia de eventos han mostrado que apneas y RGE raramente ocurren en forma simultánea y cuando ellos lo hacen, parece más bien ser eventos al $\operatorname{azar}^{29,30}$. En un estudio retrospectivo de lactantes con RGE y apnea pero no evento con riesgo inminente de muerte (ALTE), 105 lactantes con apnea recurrente se estudiaron con pHmetría de $24 \mathrm{~h}$, siendo $48 \%$ positivo para RGE, y $42 \%$ asintomáticos. La incidencia de apneas fue similar al de la población normal ${ }^{31}$. En otro estudio, 20 lactantes con ALTE y RGE clínico se estudiaron con PSG y pHmetría nocturna. Este estudio reveló un total de 334 apneas centrales y 36 apneas obstructivas. En dicho estudio los movimientos y los despertares precedieron las caídas de $\mathrm{pH}$ en $50 \%$ de los episodios de reflujo. No hubo correlación entre los parámetros de reflujo y el número o duración de las apneas ${ }^{32}$. ALTE en 130 lactantes consecutivos se evaluó en un protocolo prospectivo con PSG y pHmetría nocturna; 49 lactantes (38\%) requirieron resucitación cardiopulmonar. El RGE estuvo presente en 34 (69\%) pero sólo en 8 de ellos (24\%) se obtuvo una PSG anormal 33 . Recientemente Arad-Cohen y cols ${ }^{34}$ mostraron sólo $6,4 \%$ de eventos apneicos siguiendo a un 
episodio de reflujo, mientras que $81 \%$ de los episodios apneicos no tuvieron ninguna correlación con reflujo. En ninguno de los estudios mencionados se analizó la variable intensidad del RGE, de acuerdo a los valores actualmente aceptados, con la presencia de eventos respiratorios.

Los hallazgos inconsistentes en los estudios descritos pueden ser explicados, en parte, porque los cambios en el pH esofágico pueden no ser el evento gatillante de la apnea del lactante. En un estudio reciente, 22 lactantes se estudiaron simultáneamente con pHmetría e impedanciometría (IMP), una técnica que puede detectar cambios volumétricos en el esófago, mostrando que solamente $11 \%$ de los episodios de reflujo por IMP se detectaron por pHmetría. Más aún, el porcentaje de apneas relacionado a episodios de reflujo aumentó de 6 a 30\% cuando IMP fue adicionado a la $\mathrm{PSG}^{35}$, sugiriendo que el reflujo no ácido puede tener un rol en la apnea asociada a reflujo. Sin embargo, son necesarios mayores estudios que corroboren estos resultados iniciales, así como demostrar el rol de un reflujo no ácido sobre cambios en la vía aérea y en los centros reguladores de la respiración.

Debido a la controversia y limitaciones en el estudio de relaciones temporales (causa-efecto)

\section{REFERENCIAS}

1. Orenstein SR, Orenstein DM. Gastroesophageal reflux and respiratory disease in children. J Pediatr 1988; 112: 847-58.

2. Comes H, LALiemand P. Infant apnea and gastroesophageal reflux. Pediatr Radiol 1992; 22: 8-11.

3. RAMET J. Cardiac and respiratory reactivity to gastroesophageal reflux: experimental data in infants. Biol Neonate 1994; 65: 240-6.

4. Simpson H, Hampton F. Gastrooesophageal reflux and the lung. Arch Dis Child 1991; 66: 277-83.

5. See CC, Newman LT, Berezin S, Giassman MS, Medow MS, Dozor AJ ET AL. Gastroesophageal refluxinduced hypoxemia in infants with apparent lifethreatening events. Am J Dis Child 1989; 143: 951-4.

6. Boyle JT, Tuchman DN, Altschuler SM, Nixon TE, PACK AI, CoHen S. Mechanisms for the association of gastroesophageal reflux and bronchospasm. Am Rev Respir Dis 1985; 131: S16-20. entre cambios de $\mathrm{pH}$ y eventos respiratorios, el presente trabajo se enfocó en la búsqueda de diferencias en las características polisomnográficas de los niños, separados de acuerdo a la intensidad del RGE del punto de vista pHmétrico. En relación a los objetivos establecidos en la introducción, nuestro estudio demostró que la intensidad del RGE afecta la arquitectura del sueño, aumentando el porcentaje de sueño activo en desmedro del sueño quieto. $\mathrm{Si}$ bien, tradicionalmente se ha asociado RGE con pausas obstructivas, nuestro trabajo, al igual que los ya comentados, no encontró una relación significativa entre ambos. Más aún, la presencia de pausas y apneas centrales fue mayor en los lactantes con RGE más intenso, lo que sugiere que hay una relación entre la maduración de los centros reguladores de la respiración y el normal desarrollo de los mecanismos de control del RGE, ya sea a nivel central o de efectores. Por tanto, si bien el uso rutinario clínico de la PSG no ha permitido encontrar una relación temporal entre caída de $\mathrm{pH}$ y pausa/ apnea, la detección de RGE de mayor intensidad puede ser considerada como un factor de riesgo adicional para el desarrollo de apneas/pausas centrales, alertando al clínico a considerar un manejo oportuno del RGE.

7. Strolo PJ, Rogers RM. Obstructive sleep apnea. N Engl J Med 1996; 334: 99-104.

8. Kerr P, Shoenut JP, Milat T, Buckie P, Kryger MH. Nasal CPAP reduces gastroesophageal reflux in obstructive sleep apnea syndrome. Chest 1992; 101: 1539-44.

9. Shoenut JP, KerR P, Micflikier AB, Jamashiro $Y$, KRYGER MH. The effect of nasal CPAP on nocturnal reflux in patients with aperistaltic esophagus. Chest 1994; 106: 738-41.

10. KerR P, SHOENut J. Nasal continuous positive airway pressure. A new treatment for nocturnal gastroesophageal reflux? J Clin Gastroenterol 1993; 17: 276-80.

11. Harris $\mathrm{P}$, Muñoz C, Mobarec S, Brockmann P, Mesa $\mathrm{T}$, SÁnchez I. Relevance of the $\mathrm{pH}$ probe in sleep study analysis in infants. Rev Chile Pediatr 2001; 72(S): 10.

12. Working Group of the European Society of Pediatric Gastroenterology and Nutrition. A standandized protocol for the methodology of esophageal 
$\mathrm{pH}$ monitoring and interpretation of the data for the diagnosis of gastroesophageal reflux. J Pediatr Gastroenterol Nutr 1992; 14: 467-71.

13. Coletis $R$, Christie $D$, Orenstein $S$. Statement of the North American Society for Pediatric Gastroenterology and Nutrition. Indications for Pediatric Esophageal pH Monitoring. J Pediatr Gastroenterol Nutr 1995; 21: 253-62.

14. Arana A, Bagucka B, Hauser B, Hegar B, Urbain D, KAUfMAN L ET AL. PH monitoring in the distal and proximal esophagus in symptomatic infants. J Pediatr Gastroenterol Nutr 2001; 32: 259-64.

15. Marcus C, Omun K, Basinki D, Bailey S, Keens T, DAVIDSON S. Normal polysomnographic values for children and adolescents. Am Rev Respir Dis 1992; 146: 1235-9.

16. American Thoracic Society. Standards and indications for cardiopulmonary sleep studies in children. Official statement. Am J Respir Crit Care Med 1996; 153: 866-78.

17. Hoppenbrouwers T, Hodgman J, Harper R, HofmanN E, Stremanm M, McGinty D. Polygraphic studies of normal infants during the first six months of life: III. Incidence of apnea and periodic breathing. Pediatrics 1977; 60: 418-25.

18. Shannon D, Carley D, Kelly D. Periodic breathing: Quantitative analysis and clinical description. Pediatr Pulmonol 1988; 4: 98-102.

19. Orenstein S, Izadnia F, Khan S. Gastroesophageal reflux disease in children. Gastroenterol Clin North Am 1999; 4: 947-69.

20. Тнасн BT. Reflux associated apnea in infants: evidence for a laryngeal chemoreflex. Am J Med 1997; 103: 120-4.

21. Sitzer A, Boyle J, Tuchman D, Fox W. Awake apnea associated with gastroesophageal reflux: a specific clinical syndrome. J Pediatr 1984; 104: 200-5.

22. SACRE L, VANDEPLAS Y. Gastroesophageal reflux associated with respiratory abnormalities during sleep. J Pediatr Gastroenterol Nutr 1989; 9: 28-33.

23. Marino A, Assing E, Carbone M, Hiatt I, Hegyi T, GrafF M. The incidence of gastroesophageal reflux in preterm infants. J Perinatol 1995; 15: 369-71.

24. Menon AP, Schefrt GL, Thach BT. Apnea associated with regurgitation in infants. J Pediatr 1985; 106: 625-9.
25. Harding R, Johnson P, McCleliand ME. Liquidsensitive laryngeal receptors in the developing sheep, cat and monkey. J Physiol 1978; 277: 40922.

26. Storey AT, Johnson P. Laringeal water receptors initiating apnea in the lamb. Exp Neurol 1975; 47: 42-55.

27. Jefrerey HE, RahiLy P, Read DJ. Multiple causes of asphyxia in infants at high risk for sudden infant death. Arch Dis Child 1983; 58: 92-100.

28. Rosen CL, Frost J, HaRRison G. Infant apnea: Polygraphic studies and follow-up monitoring. Pediatrics 1983; 71: 731-6.

29. Walsh J, Farrell M, Keenan W, Lucas M, Framer M. Gastroesophageal reflux in infants: Relation to apnea. J Pediatr 1981; 99: 197-201.

30. Ariagno R, Guileminault C, Baldwin R, OwenBOEDDIKER M. Movement and gastroesophageal reflux in awake term infants with near miss» SIDS, unrelated to apnea. J Pediatr 1984; 100: 894-7.

31. Sheikh S, Stephen T, Sisson B. Prevalence of gastroesophageal reflux in infants with recurrent brief apneic episodes. Can Resp J 1999; 6: 401-4.

32. Kahn A, Rebuffat E, Sottiaux M, Blum D, Yasik E. Sleep apneas and acid esophageal reflux in control infants and in infants with an apparent life-threatening event. Biol Neonate 1990; 57: 1449.

33. Veereman-Wauters G, Bochner A, Van Caime-BerTRAND M. Gastroesophageal reflux in infants with a history of near-miss sudden infant death. J Pediatr Gastroenterol Nutr 1991; 12: 319-23.

34. Arad-Cohen N, Cohen A, Tirosh E. The relationship between gastroesophageal reflux and apnea in infants. J Pediatr 2000; 137: 321-6.

35. Wenzl T, Silny J, Schenke S, Peschgens T, Heimann G, SkopNIK H. Gastroesophageal reflux and respiratory phenomena in infants: status of the intraluminal impedance technique. J Pediatr Gastroenterol Nutr 1999; 28: 423-8.

Agradecimientos:

Los autores agradecen a los médicos, enfermeras y tecnólogas del Centro de Estudios Médicos del Sueño de la Pontificia Universidad Católica de Chile por su apoyo y asistencia técnica. 